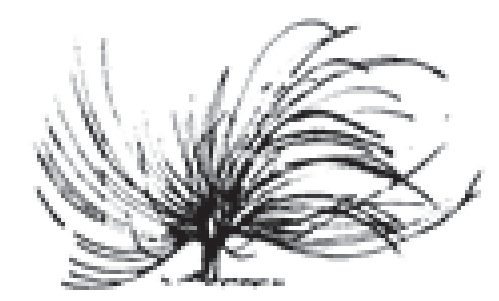

\title{
Análisis de los Sistemas de Planeación Estratégica como Soporte Académico para la Carrera de Manejo de Recursos Naturales de la Universidad Estatal a Distancia
}

\author{
Rosita Ulate Sánchez ${ }^{1}$ \\ Universidad Estatal a Distancia \\ San José, Costa Rica \\ rositaulate@gmail.com
}

\begin{abstract}
Resumen
En el presente artículo se describe un estudio de carácter exploratorio y cualitativo con un análisis comparativo documental de la declaratoria de la misión de la Universidad Estatal a Distancia (UNED), la Escuela de Ciencias Exactas y Naturales (ECEN), así como de la Carrera de Manejo de Recursos Naturales (MARENA). El propósito fue conocer la correspondencia entre los objetivos de desempeño plantados por cada uno de los niveles jerárquicos institucionales mencionados a la luz de la misión de las partes involucradas. Se utilizó para la revisión de los documentos consultados, el modelo de análisis de sistemas de gestión propuesto por Kaufman, Watkins y Leigh (2001), Organizatoinal Element Model (OEM), en donde se examinan las acciones de los niveles Mega, Macro, Micro y operacional de una organización
\end{abstract}

Recibido: 17 de setiembre de 2013-Aprobado: 25 de octubre de 2014

1 Rosita Ulate realizó sus estudios de pr grado en la Carrera de Administración de Empresas, así como su Maestría en esta misma rama de la administración con Énfasis en Mercadeo en la Fundación de Estudios de Pos grado de la Universidad de Costa Rica; actualmente es egresada del Doctorado en Educación a Distancia en Tecnología Instruccional de NOVA Southeastern University en Florida, Estados Unidos. 
educativa. Este tipo de herramientas administrativas permite visualizar la brecha existente entre lo que estipula en la misión de los niveles gerenciales y lo que se realiza en los subsiguientes estratos jerárquicos de la organización. Adicionalmente, se incrementó el estudio con una comparación usando el cotejo de las Fortalezas, Oportunidades, Debilidades y Amenazas (FODA), para así identificar las posibles acciones de cambio en la gestión de la planeación a nivel institucional hacia una cultura de vinculación docencia, extensión e investigación. Los hallazgos encontrados evidenciaron una serie de debilidades en las propuestas de la gestión académica y administrativa con respecto a las bases de la planeación estratégica, como lo es la declaratoria de una misión. Con lo que se marcan el requerimiento de toma de decisiones y cambios estructurales que afectan la calidad de los procesos de enseñanza y aprendizaje del programa en estudio de la carrera de Manejo de Recursos Naturales.

Palabras clave: gestión académica y administrativa, toma de decisiones, niveles jerárquicos institucionales, planeación estratégica

\begin{abstract}
This article describes an exploratory qualitative study with a comparative documental analysis of the mission statement of Distance State University of Costa Rica (UNED, for its acronym in Spanish), the School of Exact and Natural Sciences as well as the major of Naural Resource Management. The purpose was to know the correspondence between the learning objectives stated in each of the institutional hieararchical levels in light of the mission statements of the parties involved. To review the consulted documents, the researcher used theOrganizatoinal Element Model (OEM) proposed by Kaufman, Watkins and Leigh (2001), in which the actions of the mega, macro, and micro and operational levels of an educational organization are examined. This type of administrative tools make it possible to visualize the existing gap between what is stated in the mission statement of the managerial levels and what is done in the subsequent
\end{abstract}


hierarchical levels of the organization. Additionally, the study was complemented by a comparison of the Strengths, Weaknesses, Opportunities, and Threats (SWOT) of the organization to indentify possible actions to bring about change in the planning management towards a culture that links teaching, outreach, and research. The findings evidence a series of weaknesses in the academic and administrative management proposals with regard to the basis for strategic plannic such as stating a mission, which deals with decision-making and structural changes that affect the quality of the teaching/learning processes in the program of the manjor of Natural Resource Management.

Keywords: academic and administrative management, decision-making, institutional hierarchical levels, strategic planning

\section{Introducción}

L

a planificación estratégica es un marco de trabajo que permite a una organización alinear sus recursos y esfuerzos en la consecución de su visión, misión, objetivos y metas en el corto, mediano y largo plazo.

Son muchas las herramientas de análisis de la planeación aportadas por las ciencias económicas en los procesos administrativos. Sin embargo, para efectos de este estudio se tomó el Modelo de los Elementos Organizacionales (Organizatoinal Element Model- OEM) propuesto por Kaufman, Watkins y Leigh (2001), especialmente usado en el ámbito institucional educativo. El principal aporte de este enfoque sistémico (sistema lógico de solución de problemas y necesidades), OEM es el detectar descriptivamente la brecha de lo que se promete hacer $y$ de lo que se hace en una entidad académica. Para Kaufman, Watkins y Leight (2001), los sistemas organizacionales están integrados por otros subsistemas distribuidos en al menos cuatro niveles jerárquicos, entre ellos Mega, Macro, Micro y Operacional (pudiendo agregar niveles de integración hacia lo Supra Mega y Supra Micro, dependiendo de cada caso a valorar). Los sistemas y sub-sistemas funcionan integral e interrelacionalmente en cada uno de los procesos de planeación, organización, dirección y control de sus funciones y propósitos. 
Una institución académica de enseñanza superior responde a las exigencias de la sociedad en la formación de nuevos profesionales. Como contribución la gestión académica, la planeación estratégica, de manera secuencial, impregna la misión planteada en cada uno de los niveles jerárquicos existentes y alinea todos los esfuerzos y recursos a la consecución de los objetivos determinados y expuestos al público.

\section{Metodología}

La investigación realizada fue de carácter cualitativa con un diseño exploratorio, descriptivo que utilizó el análisis sistémico OEM. La unidad de análisis es la carrera en estudio: el Programa de Protección y Manejo de Recursos Naturales (MARENA) antes conocido como PROMARENA.

Se tomó en cuenta la experiencia empírica de la autora en los puestos de asistencia académica de la Dirección de la Escuela de Ciencias Exactas y Naturales (ECEN) del 2008 al 2011. Adicionalmente se realizaron entrevistas a profundidad a funcionarios académicos con cargos de autoridad dentro de la ECEN para conocer sus percepciones y las funciones realizadas. El instrumento utilizado fue una guía temática no estructurada de preguntas abiertas.

Como fuentes secundarias se recurrió a la consulta de varios documentos internos de la universidad, tales como: a) Informe de Análisis y Diseño de Sistema/ITDE 8006 (Ulate, 2009); b) Informe de Autoevaluación del Programa de Protección y Manejo de Recursos Naturales (ECEN, 2008) y Debilidades Comunes de las Carreras Autoevaluadas en la Escuela de Ciencias Exactas y Naturales: Posibles Causas y Niveles Institucionales para la Solución (Azze, 2009).

Las declaraciones de la misión de cada uno de los niveles jerárquicos analizados fueron expuestos en un esquema que permitió conocer por categorías los compromisos definidos en sus declaratorias oficiales.

La información recolectada fue organizada, categorizada y procesada bajo los conceptos teóricos de Kaufman, Watkins y Leigh (2001):

1) Establecer los niveles (Supra Mega, Mega, Macro, Micro, Supra Micro), tomando como nivel Macro la carrera en estudio.

2) Describir las principales características del nivel Macro. 
3) Comparar la declaración de misión de los niveles Mega (Escuela), Macro (Carrera) y Micro (cátedras, profesores y estudiantes).

4) Desglosar las metas y principales actividades de los niveles en estudio.

5) Identificar las brechas existentes entre la misión y visión de los niveles Mega, Macro y Micro.

6) Mencionar las necesidades de atención en la planeación estratégica de los niveles estudiados.

A partir de los resultados obtenidos con el modelo OEM, se procedió a un análisis FODA para luego presentar una serie de acciones posibles de realizar como plan de mejora en la gestión de planeación estratégica.

Se procesó la información y se realizaron resúmenes expuestos por medio de esquemas para su respectivo análisis, todos desarrollados en las herramientas de Office Windows ${ }^{\circledR}$, específicamente los programas Word® y Excell®.

La principal limitante del estudio fue la no existencia de un plan estratégico en el nivel de las cátedras de la carrera, por lo cual no se logró concretar una comparación total entre los niveles.

\section{Antecedentes}

La propuesta académica de educación a distancia ofrecida por la UNED responde al mandato gubernamental de Costar Rica de "ofrecer educación superior a todos los sectores de la población, especialmente, a aquellos que por razones económicas, sociales, geográficas, culturales, etarias, de discapacidad o de género, requieran oportunidades para una inserción real y equitativa en la sociedad (...) y de compromiso con la sociedad, y el medio ambiente" (...) se compromete con la excelencia académica, el desarrollo de la cultura, la ciencia, el arte y los derechos humanos para la construcción de una sociedad justa y una cultura de paz" (UNED, 2009).

El modelo pedagógico de la UNED está construido bajo un marco ecléctico en su propuesta educativa con aportes de las teorías del aprendizaje del conductismo, cognitivismo y el constructivismo, pero siempre con un norte del estudiante como centro del proceso de enseñanza y aprendizaje. Estas diferentes posiciones teóricas han imperado en las carreras ofrecidas por la Escuela de Ciencias Exactas y Naturales. 
La carrera de Manejo de Recursos Naturales (MARENA), antes llamada Protección y Manejo de Recursos Naturales (PROMARENA) fue creada a partir de la alianza y convenio de dos instituciones públicas (UNED y el Ministerio de Ambiente, Energía y Mares de Costa Rica- MINAE). De tal manera, que la carrera llegó a convertirse en una opción de educación superior, especialmente en poblaciones de estudiantes que se encuentran en zonas rurales relacionados con el área de conocimiento de los recursos naturales. El plan de estudios se enfocó en la formación de futuros profesionales comprometidos con el desarrollo sostenible de Costa Rica y en el mantenimiento y protección de su diversidad biológica.

\section{La Carrera de Manejo de Recursos Naturales (Nivel Macro)}

Como centro del análisis de este estudio, la carrera MARENA se ubicó en el nivel Macro, la ECEN en el nivel Mega y las cátedras con cursos del programa en el nivel Micro, manteniendo otros niveles no evaluados, como lo son la sociedad y los estudiantes, (véase Figura 1).

Figura 1. Determinación de niveles y subniveles del sistema en análisis: Carrera de Manejo de los Recursos Naturales (Macro).

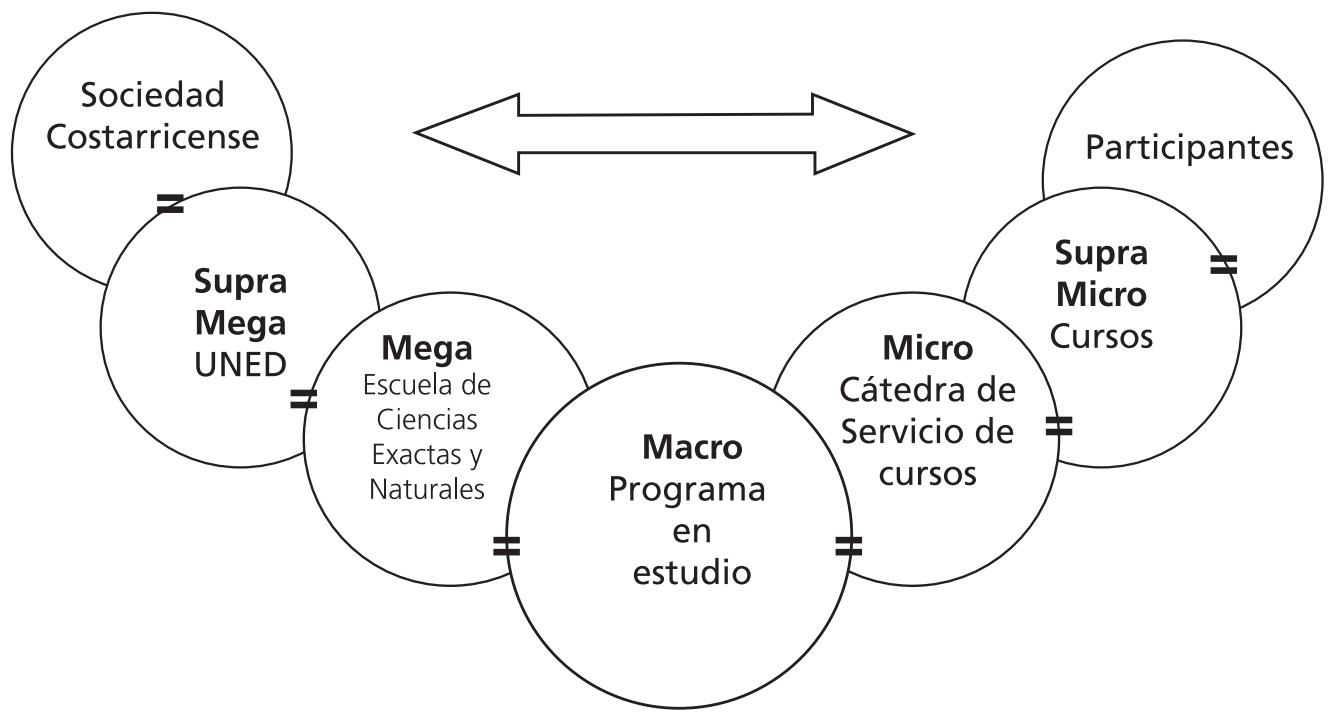


La carrera ofrece grados de bachillerato y licenciatura universitaria, para ello se apoya en cursos que son ofrecidos por varias cátedras de la Escuela de Ciencias Exactas y otros cursos de servicio a cargo de las escuelas de Administración y de Educación de la UNED. El plan de estudio hasta el 2011 estaba integrado por 52 asignaturas distribuidas en al menos cinco áreas de conocimiento (véase Figura 2).

Figura 2. Áreas de conocimiento que albergan las 52 asignaturas del plan de estudios de MARENA.

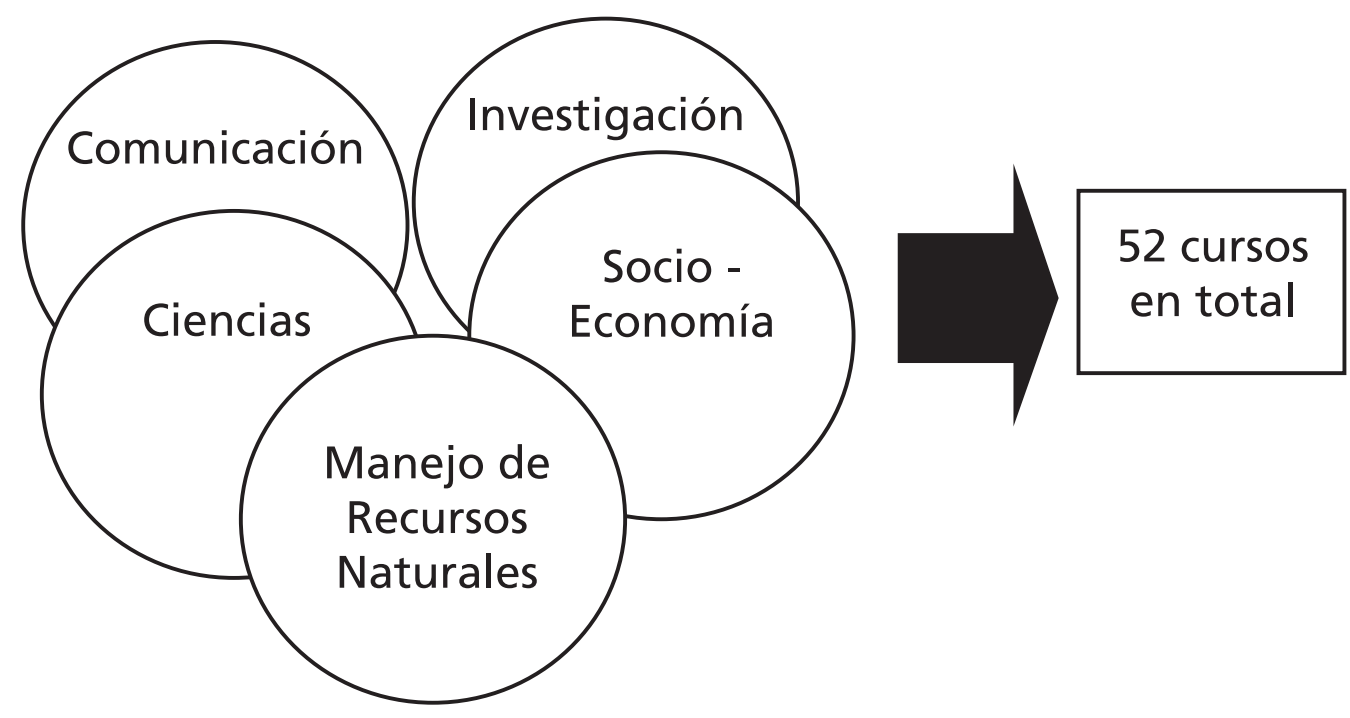

Son varios los grupos de actores sociales que participan en el nivel Macro, se encuentran en orden jerárquico funcional de la UNED: a) Encargado de Programa, b) Encargados de Cátedra; c) Personal administrativo y de asesoría académica (staff); d) profesores y finalmente e) estudiantes (véase Figura 3). 
Figura 3. Determinación de grupos de actores sociales en el nivel macro: Carrera de Manejo de Recursos Naturales (MARENA).

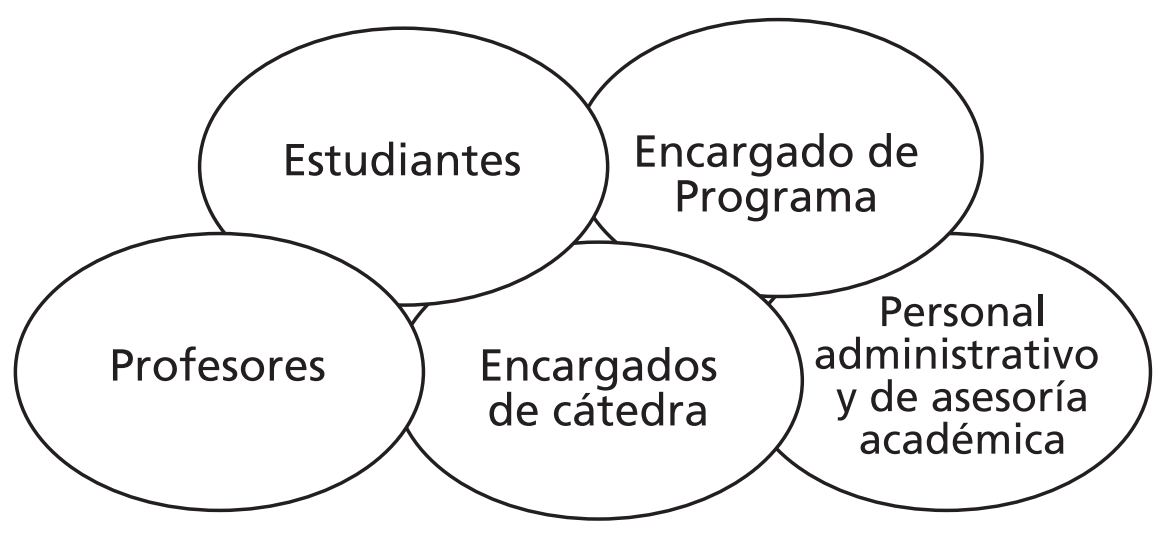

En cuanto a los estudiantes de la carrera, se llegó a determinar por medio de las fuentes secundarias consultadas, que tienen principalmente un estado civil de solteros, en su mayoría de nacionalidad costarricense, matriculados en los centros universitarios de la UNED de todo el país, con un alto porcentaje de matrícula de zonas rurales de Costa Rica (UNED, 2008).

El programa en estudio se desarrolla con un enfoque de enseñanza de educación a distancia con cursos híbridos (comunicación sincrónica y asincrónica). Entre las estrategias de acompañamiento al estudiante se ofrecen tutorías presenciales, materiales didácticos impresos, multimediales, soporte en línea, videoconferencias y una significativa realización de actividades de aprendizaje como laboratorios, prácticas y giras de campo.

\section{Comparación OEM de los compromisos de la misión por niveles jerárquicos del sistema en estudio (UNED - ECEN - MARENA)}

Se presenta a continuación una revisión del programa a través del análisis del perfil de la misión por niveles para la determinación de necesidades y brechas existentes, siempre bajo el modelo sistémico de 
Kaufman, Watkins y Leigh (2001). En la Figura 4 se describieron los principales servicios ofrecidos a la sociedad costarricense y expuestos en la misión de los niveles estudiados.

En la misma Figura 4, en el primer nivel se expone la misión de la institución, a partir de la cual se deducen varias categorías de criterios de planeación estratégica: a) educación superior a toda la población; b) el uso de medios tecnológicos y que promuevan el aprendizaje independiente; c) compromiso con la sociedad y el medio ambiente; d) enfoques de formación humanista, cultura, ciencia, arte, derechos, justicia y cultura de paz y finalmente e) compromiso de excelencia académica.

Seguidamente en la misma figura 4, en el segundo nivel se expone la misión de la Escuela de Ciencias Exactas, de donde se extraen sus compromisos generales tales como: a) formación profesional en áreas de las ciencias exactas y naturales; b) ofrecimiento de una modalidad de educación a distancia; c) formación de profesionales que respondan a las necesidades del entorno; d) un eje de vinculación docencia, extensión y extensión; e) promoción del empredurismo, razonamiento crítico, ética, valores de sostenibilidad, equidad y calidad de vida; f) promover un modelo curricular ajustado a las necesidades del mercado y finalmente g) contar con un personal altamente calificado.

Más abajo en la misma figura 4, se presenta la misión de la carrera en estudio y se extraen cuatro compromisos básicos: a) formación de profesionales en protección, preservación y restauración de los recursos naturales; b) promoción de la capacidad para transferir conocimientos y nuevas tecnologías eficientes; c) consideración de la realidad económica y sociocultural en su plan de estudios y d) cooperación con el desarrollo sustentable del país.

Un poco más abajo en la figura 4 se logra determinar que otro de los niveles, en este caso el de las cátedras (encargado de cátedra, profesores) no se evidencia una declaración de misión, sin embargo si se logra conocer sus funciones dentro del proceso académico tales como: a) ofrecer cursos al programa de estudios de las carreras; b) promoción de actividades teórico-prácticos; c) atención de asignaturas por áreas de estudio y d) desarrollo de diseños curriculares para los cursos ofrecidos en los programas de estudio. 


\section{Evidencia del análisis por niveles}

Conforme se presentan los diferentes niveles se observan cuatro compromisos en el nivel Supra Mega de la institución, siete compromisos en el nivel Mega, la Escuela de Ciencias Exactas y Naturales; cuatro compromisos en el nivel Macro, Carrera de Manejo de Recursos Naturales y finalmente en el último nivel Micro, de las Cátedras de las asignaturas, otros cuatro compromisos.

Al alinear los compromisos por similitud de categorías de criterios en la figura 4, se observa que hay espacios que no han sido considerados en los niveles previos y subsiguientes del Macro (como es el caso del ítem 4 del nivel Macro), denominado como vinculación docencia, investigación y extensión.

En vista de que no se encontró misión o visión en el nivel Micro (cátedras con cursos para la carrera), se procedió a un análisis de las tareas funcionales que si se realizaron en el periodo de estudio. El propósito siempre fue verificar el cumplimiento de los propósitos planteados en la misión de los niveles Mega y Macro. Se encontraron las siguientes tareas agrupadas por categorías de trabajo, (véase Figura 5):

1. Tareas en la oferta de los cursos: generar diseños curriculares, oferta de cursos, producción de materiales, seguimiento de matrícula, planificar tutorías, prácticas y laboratorios, evaluación de los aprendizajes.

2. Actividades didácticas: teórico- prácticas, estudio individual, laboratorios, giras de campo, videoconferencias, asignación de investigaciones.

3. Proyectos de investigación y extensión: sólo en dos cátedras.

4. Áreas de conocimiento y ejes transversales: están divididas y se respetan los ejes transversales.

5. Diseños curriculares: para cada curso con su propósito, objetivos, temas, estrategias de aprendizaje, evaluación, plantilla de actividades en la plataforma de aprendizaje.

6. Dirección de recursos humanos: contratación y capacitación de profesores. 
Análisis de los Sistemas de Planeación Estratégica como Soporte Académico para la Carrera de Manejo de Recursos Naturales de la

\section{Figura 4. Análisis del perfil de misión por niveles Mega, Macro y Micro (Adaptación de Kaufman et al., 2001, p.107).}

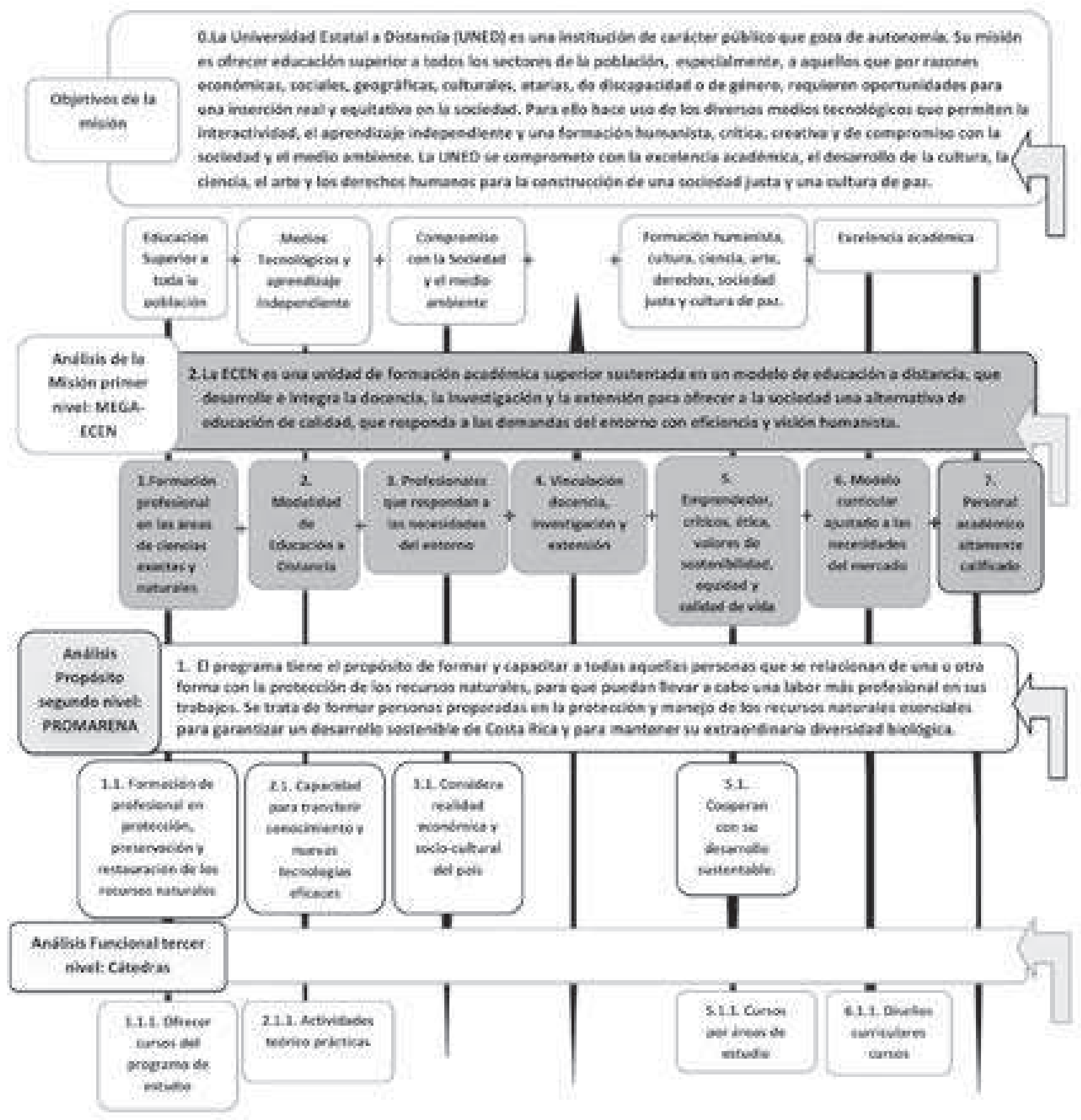


Figura 5. Análisis de las tareas realizadas en el Micro: Cátedras de MARENA en la ECEN.

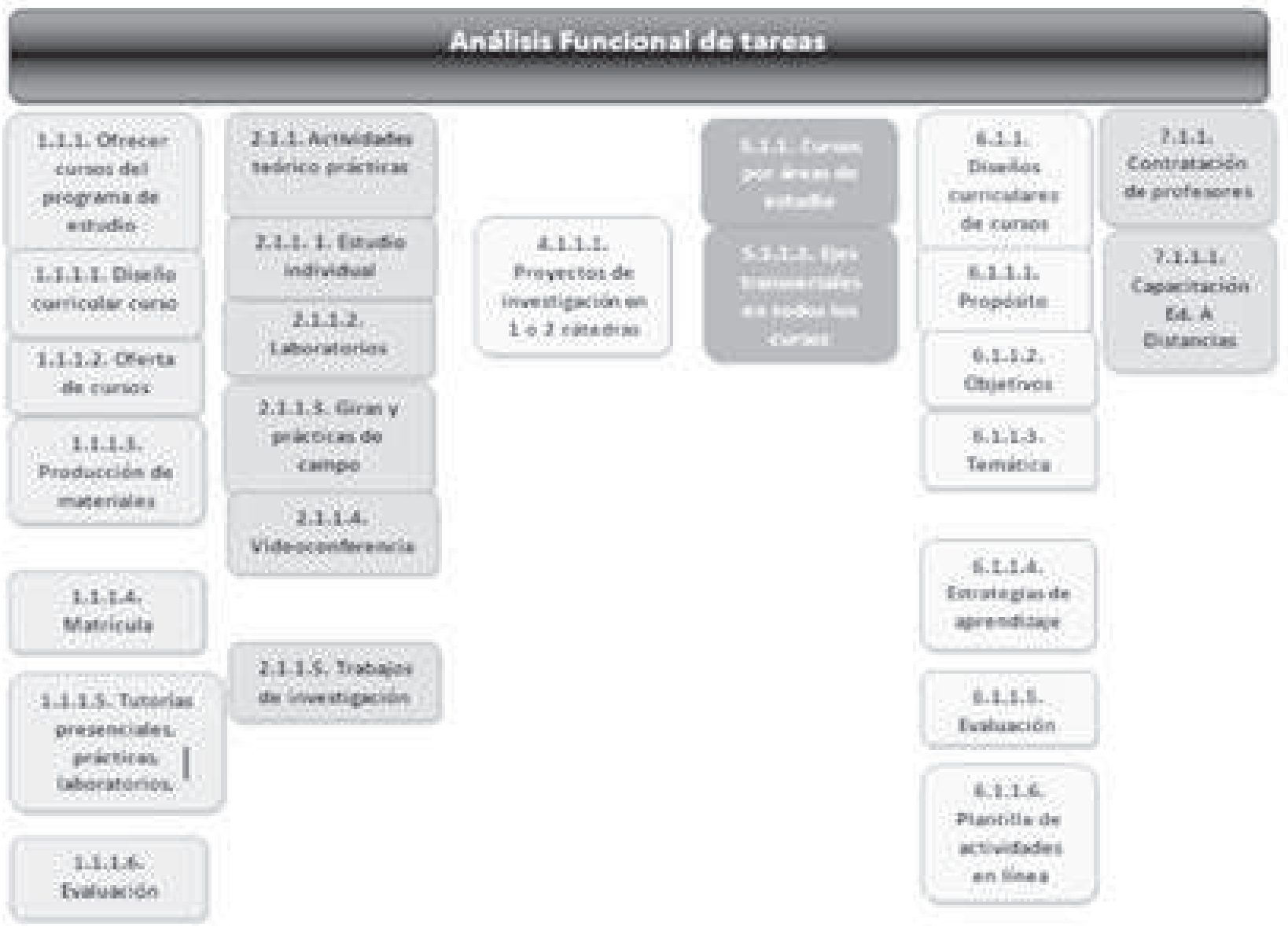

\section{Análisis FODA}

Adicionalmente se agregó al modelo OEM un análisis FODA de la carrera de Manejo de Recursos Naturales (MARENA), con el fin único de contar e integrar mayor información posible de obtener de cada herramienta utilizada (véase Tabla 1). 
Análisis de los Sistemas de Planeación Estratégica como Soporte

Académico para la Carrera de Manejo de Recursos Naturales de la

Universidad Estatal a Distancia

Tabla 1: Análisis FODA del programa en estudio

\begin{tabular}{|c|c|c|c|}
\hline Fortalezas & Debilidades & Oportunidades & Amenazas \\
\hline $\begin{array}{l}\text { Carrera innovadora } \\
\text { en el país }\end{array}$ & $\begin{array}{l}\text { Organización matricial edu- } \\
\text { cativa Programas y Cátedras. } \\
\text { Impide identificación, comu- } \\
\text { nicación y realimentación* }\end{array}$ & $\begin{array}{l}\text { Tendencia en conser- } \\
\text { vación de zonas }\end{array}$ & $\begin{array}{l}\text { Apertura de carreras } \\
\text { afines }\end{array}$ \\
\hline $\begin{array}{l}\text { Responde a polí- } \\
\text { ticas de conserva- } \\
\text { ción ambiental }\end{array}$ & $\begin{array}{l}\text { Falta de incorporación de las } \\
\text { TIC* }\end{array}$ & $\begin{array}{l}\text { Estudio de Bench- } \\
\text { marking en el país }\end{array}$ & $\begin{array}{l}\text { Falta de acceso de estu- } \\
\text { diantes a las TIC en zo- } \\
\text { nas rurales }\end{array}$ \\
\hline $\begin{array}{l}\text { Modelo Educación } \\
\text { a Distancia }\end{array}$ & $\begin{array}{l}\text { Canales de comunicación en- } \\
\text { tre el nivel macro y micro* }\end{array}$ & $\begin{array}{l}\text { Proyección social en } \\
\text { el país de la carrera* }\end{array}$ & $\begin{array}{l}\text { Mercado laboral con } \\
\text { mejores salarios }\end{array}$ \\
\hline $\begin{array}{l}\text { Plataforma } \quad \text { de } \\
\text { aprendizaje } \\
\text { nible }\end{array}$ & $\begin{array}{l}\text { Falta de sistematización de } \\
\text { procesos e información en el } \\
\text { área.* }\end{array}$ & $\begin{array}{l}\text { Integración docencia, } \\
\text { investigación y exten- } \\
\text { sión* }\end{array}$ & $\begin{array}{l}\text { Cambio constante tec- } \\
\text { nológico. Brecha digital } \\
\text { existente }\end{array}$ \\
\hline $\begin{array}{l}\text { Autoevaluación y } \\
\text { actualización del } \\
\text { programa }\end{array}$ & $\begin{array}{l}\text { Falta plan de desarrollo per- } \\
\text { sonal académico* }\end{array}$ & $\begin{array}{l}\text { Actividades de actua- } \\
\text { lización }\end{array}$ & $\begin{array}{l}\text { Restricción en fondos } \\
\text { para el desarrollo acadé- } \\
\text { mico (presupuesto uni- } \\
\text { versidades públicas) }\end{array}$ \\
\hline $\begin{array}{l}\text { Actualización de } \\
\text { materiales }\end{array}$ & $\begin{array}{l}\text { Falta plan de evaluación des- } \\
\text { empeño*. Tiempo de produc- } \\
\text { ción interna. Falta de incenti- } \\
\text { vos económicos en la paga de } \\
\text { producción }\end{array}$ & $\begin{array}{l}\text { Promoción de la Ca- } \\
\text { rrera*. Productores } \\
\text { propios, internos }\end{array}$ & $\begin{array}{l}\text { Falta de autores en el } \\
\text { mercado vs. paga de } \\
\text { producción. }\end{array}$ \\
\hline $\begin{array}{l}\text { Diseño de nuevos } \\
\text { cursos y rediseño } \\
\text { de cursos }\end{array}$ & $\begin{array}{l}\text { No hay planeación estratégi- } \\
\text { ca por debajo del nivel ma- } \\
\text { cro* Falta de vinculación }\end{array}$ & $\begin{array}{l}\text { Asignación de cargas } \\
\text { académicas para in- } \\
\text { vestigación y exten- } \\
\text { sión }\end{array}$ & $\begin{array}{l}\text { Tendencias en el área de } \\
\text { estudio }\end{array}$ \\
\hline \multirow[t]{3}{*}{$\begin{array}{l}\text { Producción de ma- } \\
\text { teriales con media- } \\
\text { ción pedagógica }\end{array}$} & $\begin{array}{l}\text { Falta de diseños didácticos } \\
\text { (instruccionales) con especi- } \\
\text { ficaciones específicas de las } \\
\text { actividades de aprendizaje* } \\
\text { Falta de análisis de resulta- } \\
\text { dos. }\end{array}$ & $\begin{array}{l}\text { Flexibilidad curricu- } \\
\text { lar. Implementación } \\
\text { de diseño didáctico } \\
\text { (instruccional). }\end{array}$ & $\begin{array}{l}\text { Falta de especialistas en } \\
\text { diseño didáctico. }\end{array}$ \\
\hline & $\begin{array}{l}\text { Falta de diseño curricular } \\
\text { de actividades de aprendiza- } \\
\text { je, como giras, prácticas de } \\
\text { campo, laboratorios* }\end{array}$ & $\begin{array}{l}\text { Utilización de medios } \\
\text { modernos de ense- } \\
\text { ñanza reutilizables* }\end{array}$ & \\
\hline & $\begin{array}{l}\text { Falta de reglamentación, cri- } \\
\text { terios de calidad de las activi- } \\
\text { dades de aprendizaje* }\end{array}$ & $\begin{array}{l}\text { Mejorar sistemas de } \\
\text { comunicación estu- } \\
\text { diantes }\end{array}$ & \\
\hline
\end{tabular}

Nota: Todas las casillas en donde se indica un asterisco $(*)$ fueron consideradas en el plan de mejoras de la carrera MARENA. 


\section{Resultados y análisis}

Se identifican varias brechas, tal como se observó en la f,igura 4. En primer lugar, se conoce la no declaratoria de un modelo de vinculación docencia, investigación y extensión en el nivel Mega Supra, mientras que a nivel Mega (ECEN) y Micro (MARENA) son de vital importancia en su compromiso con la pertinencia de las carreras ofrecidas (ver puntos 4.1.1., 4.1.1.1. de las figuras 4 y 5).

En segundo lugar, la planeación estratégica académica no permea los niveles inferiores a la categoría Macro (MARENA). Existe periódicamente planes estratégicos académicos a nivel Supra Mega, Mega y Macro mientras que en el nivel Micro sólo se indican funciones y tareas. De tal manera, el nivel Micro cumple en la oferta de cursos del programa en estudio, sin una identificación y pertinencia con los lineamientos propios de cada programa de estudio. No se conoce de declaratorias del propósito (misión) de las cátedras, ni el desarrollo de estrategias que permitan la planeación de trabajo anual. Por lo tanto, se comprueba que esa misma planeación no existe en los niveles inferiores al Micro (ver puntos 4 y 5 1.1.1., 2.1.1, 3.1.1., 4.1.1, 5.1.1., 6.1.1., 7.1.1. de las figuras 4 y 5).

En el análisis FODA, se detectaron ocho aspectos de fortalezas en los temas de una carrera innovadora, con un modelo de educación a distancia; carrera que responde a las políticas del país y de la UNED en materia de conservación de los recursos naturales. Se cuenta en la UNED con acceso a la tecnología de la comunicación y de la información (TIC), en un proceso de autoevaluación y mejora de su currículo y en la producción de materiales didácticos.

En cuanto a las debilidades de la carrera se menciona una organización funcional matricial que aparentemente no genera una comunicación entre los niveles, especialmente en el Macro y el Micro en cuanto al seguimiento de la misión. No se observa continuidad de la planeación estratégica en el nivel Micro. La carrera requiere usar los recursos TIC disponibles en la institución. Se percibieron inquietudes del personal entrevistado en cuanto a la evaluación de desempeño, tiempos adecuados a las cargas académicas e incentivos económicos equitativos al mercado laboral.

Por otro lado, en cuanto a las oportunidades se mencionaron la creciente tendencia en temas de ambiente; la posible proyección social de la carrera en el país mediante proyectos de docencia, extensión e investigación; la mejora de su currículo educativo, curricular y didáctico. 
Dentro de las amenazas se mencionaron el reto constante de actualización de las TIC; la apertura de nuevas carreras afines a MARENA en Costa Rica; la existencia de la brecha digital en zonas rurales; preocupación en cuanto al presupuesto nacional y su aporte a las universidades públicas, especialmente, su repercusión en la nivelación de salarios económicos para contar con un personal calificado y de calidad profesional.

En la figura 6 se presenta un resumen de las brechas y necesidades por categorías (calidad, identificación, vinculación, uso de TIC y otros parámetros de organización) que se esperarían ser atendidas en un futuro cercano, con el objetivo de fortalecer integración de la planeación estratégica y su gestión en los tres niveles jerárquicos analizados: Mega, Macro y Micro, que corresponden a la UNED, a la ECEN y a la carrera MARENA.

\section{Figura 6. Necesidades detectadas en el análisis OEM y FODA.}

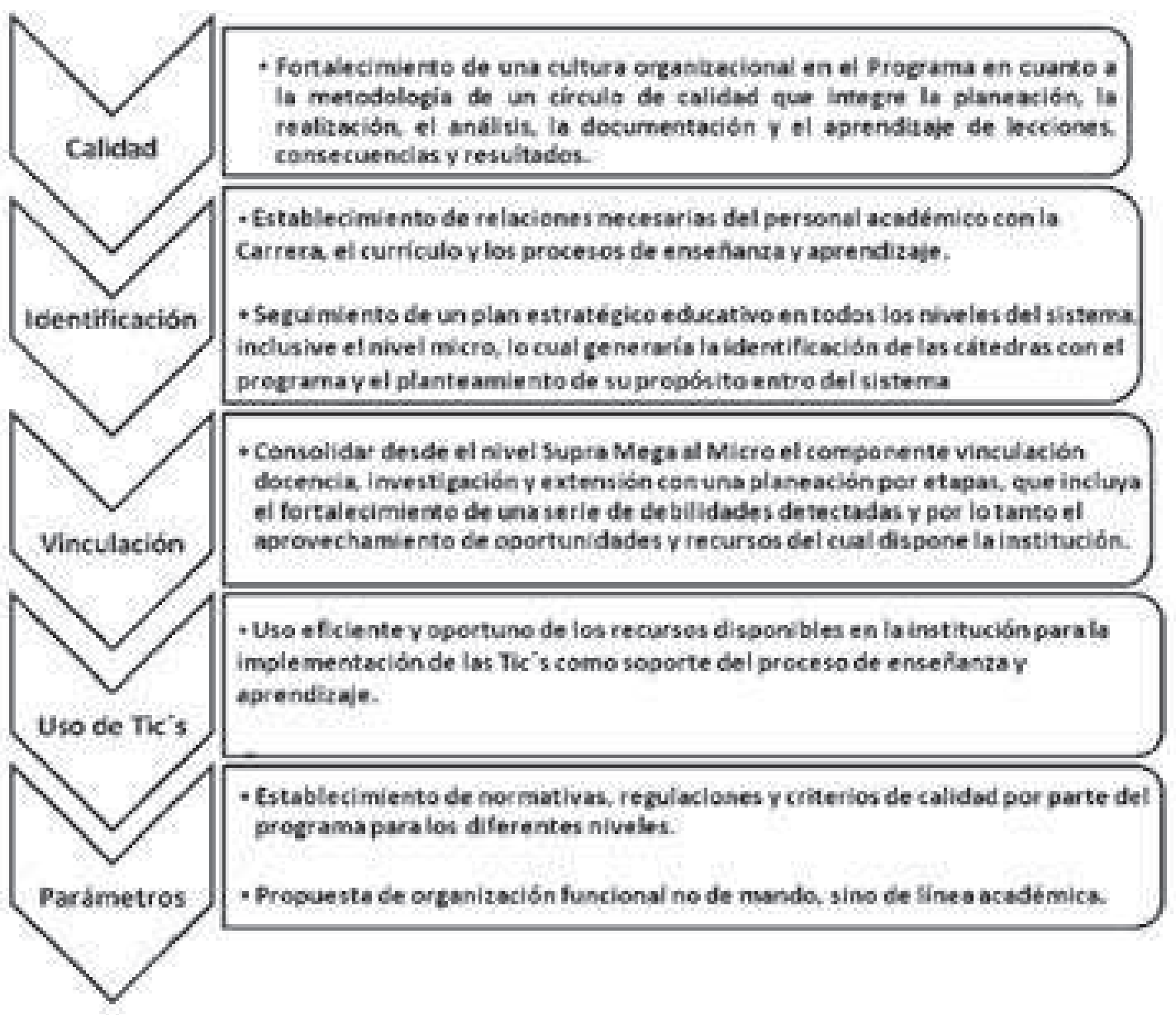




\section{Consideraciones finales y algunas recomendaciones}

Los aspectos señalados en la tabla 1 confirman la evidencia encontrada en el proceso de autoevaluación del programa de acuerdo con Azze (2009). Las debilidades estructurales son producto de la falta de una cultura organizacional de análisis y evaluación en sus procesos académicos y administrativos de forma integral. Por esa razón, los procesos carecen de una debida sistematización de experiencias y procedimientos, los cuales son necesarios para la automatización de sistemas de información como apoyo a la gestión académica y administrativa.

A partir del uso de los dos modelos de análisis se evidenció la necesidad de integrar la planeación estratégica en los niveles estudiados. La declaratoria de una misión expuesta en los medios públicos se convierte en un compromiso adquirido ante la sociedad costarricense. Para remediar esas deficiencias se proponen varias acciones prioritarias de solución:

1) Incorporación de un sistema de análisis de la correlación de planeación estratégica ${ }^{2}$ por niveles en la Institución ${ }^{3}$. La implementación de un sistema así, permite el desarrollo de una cultura organizacional de análisis y evaluación en sus procesos académicos y administrativos en todos los niveles. Un sistema de evaluación constante promueve planear, analizar, evaluar, documentar y aprender de las experiencias propias del proceso (véase figura 7).

2) La excelencia académica se logra al promover la incorporación de un sistema de calidad. Tal como se demostró en el análisis realizado en el programa de estudio en la ECEN, la actual declaratoria institucional carece de dos aspectos y acciones académicas que han sido una práctica real, durante los últimos años en la UNED, la vinculación de docencia, extensión e investigación y el creciente uso de las TIC. Ambos temas necesarios de retomar en la misión, por ende, en su actual modelo pedagógico (véase Figura 8).

2 Sistema de análisis de la gestión estratégica de planeación por correlación de los niveles Supra Mega, Mega, Macro, Micro y Operativo de toda la institución como fuente de detención de brechas desde la misión, visión, objetivos, formulación de política institucional, normativas, procedimientos y evaluación de resultados

3 Entendiendo modelo educativo a la conformación de: a) modelo pedagógico, b) modelo curricular, c) modelo didáctico (instruccional) y d) modelo informacional. 
Análisis de los Sistemas de Planeación Estratégica como Soporte Académico para la Carrera de Manejo de Recursos Naturales de la Universidad Estatal a Distancia

\section{Figura 7. Componentes de un círculo de calidad.}

\section{Necesidad: círculo de calidad del sistema}

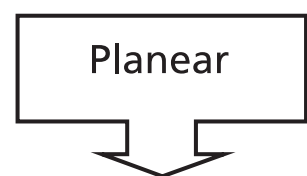

Actividades individuales, colectivas.

Considerar

objetivo, actividades, tareas, tiempo, extensión, requerimientos

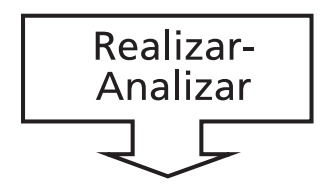

$$
\begin{gathered}
\text { Evaluar lo } \\
\text { ocurrido y } \\
\text { desarrollado en } \\
\text { el proceso. } \\
\text { Comprobar si } \\
\text { hay correlación } \\
\text { entre resultados } \\
\text { y lo deseado }
\end{gathered}
$$

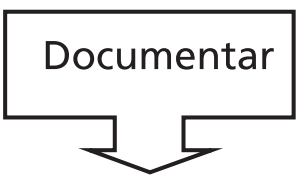

$$
\begin{aligned}
& \text { Registrar los } \\
& \text { aspectos del } \\
& \text { conocimiento y } \\
& \text { resultados para } \\
& \text { futuras } \\
& \text { decisiones }
\end{aligned}
$$

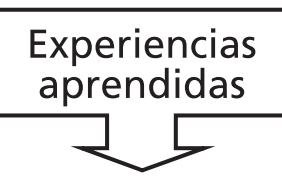

Comprobar y decidir si cada actividad proceso 0 estructura es sostenible o si requiere cambios

Nota: Adaptado de Knoll (s.f.) citado por Azze (2009).

\section{Figura 8. Vinculación Docencia, investigación y extensión de PROMARENA.}

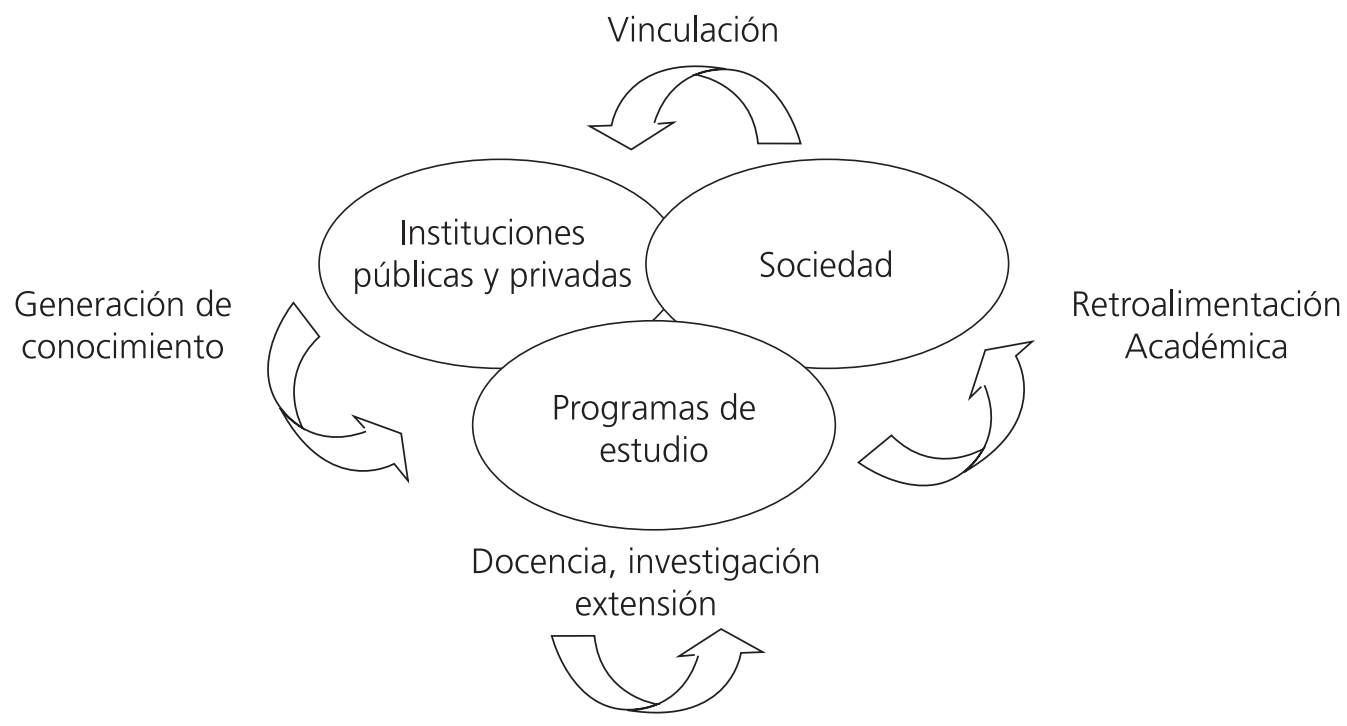

Nota: Adaptado de U.Veracruzana (2006) 
3) Integración en todos los niveles institucionales de un modelo de vinculación docencia, investigación y extensión. El compromiso social motiva el establecimiento de requisitos, recursos y acciones prioritarias en el planeamiento del sistema y los subsistemas. Esta integración conlleva varias etapas. La primera es alinear las funciones sustantivas organizativas del sistema y procesos del modelo pedagógico, al cual responderá la planeación, la organización, el establecimiento de condiciones y condicionamientos. La segunda etapa operativa es la implementación y desarrollo en respuesta a las necesidades demandadas socialmente, tales como servicios, investigación, capacitación, entre otros.

La tercera etapa es la sistematización como seguimiento, análisis y evaluación de los resultados brindando actualización a la academia, con insumos en los materiales didácticos de formación académica (véase Figura 9).

Figura 9. Etapas de la vinculación docencia, investigación y extensión de MARENA

Etapa 1

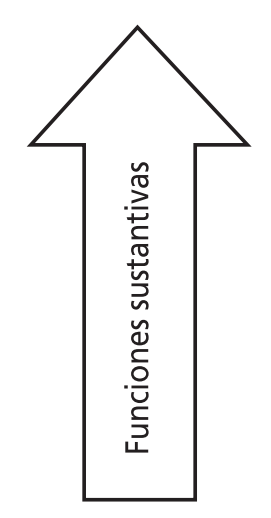

ORGANIZACIÓN

Planeación y organización Establecimiento de Condiciones y condicionantes
Etapa 2

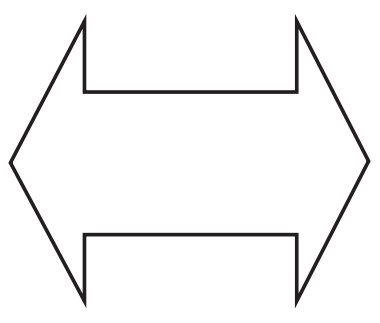

DEMANDA SOCIAL OPERATIVA

Operación, comunicación beneficios recíprocos, intercambio de saberes, participación activa
Etapa 3

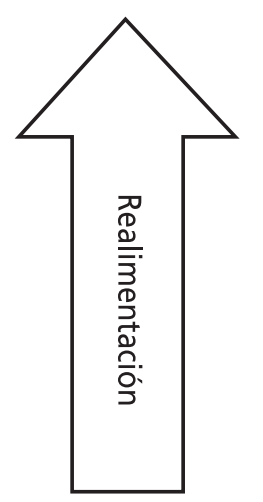

SISTEMATIZACIÓN

Seguimiento, evaluación sistematización, establecimiento de canales académicos para regresar experiencia

Nota: Adaptación de IES, Universidad Veracruzana (2006) 
La interacción de los tres ejes docencia, investigación y extensión es lo que permite a la academia la generación y aplicación de nuevos conocimientos y servicios.

\section{Figura 10. Funciones en la Vinculación de Programa en estudio}

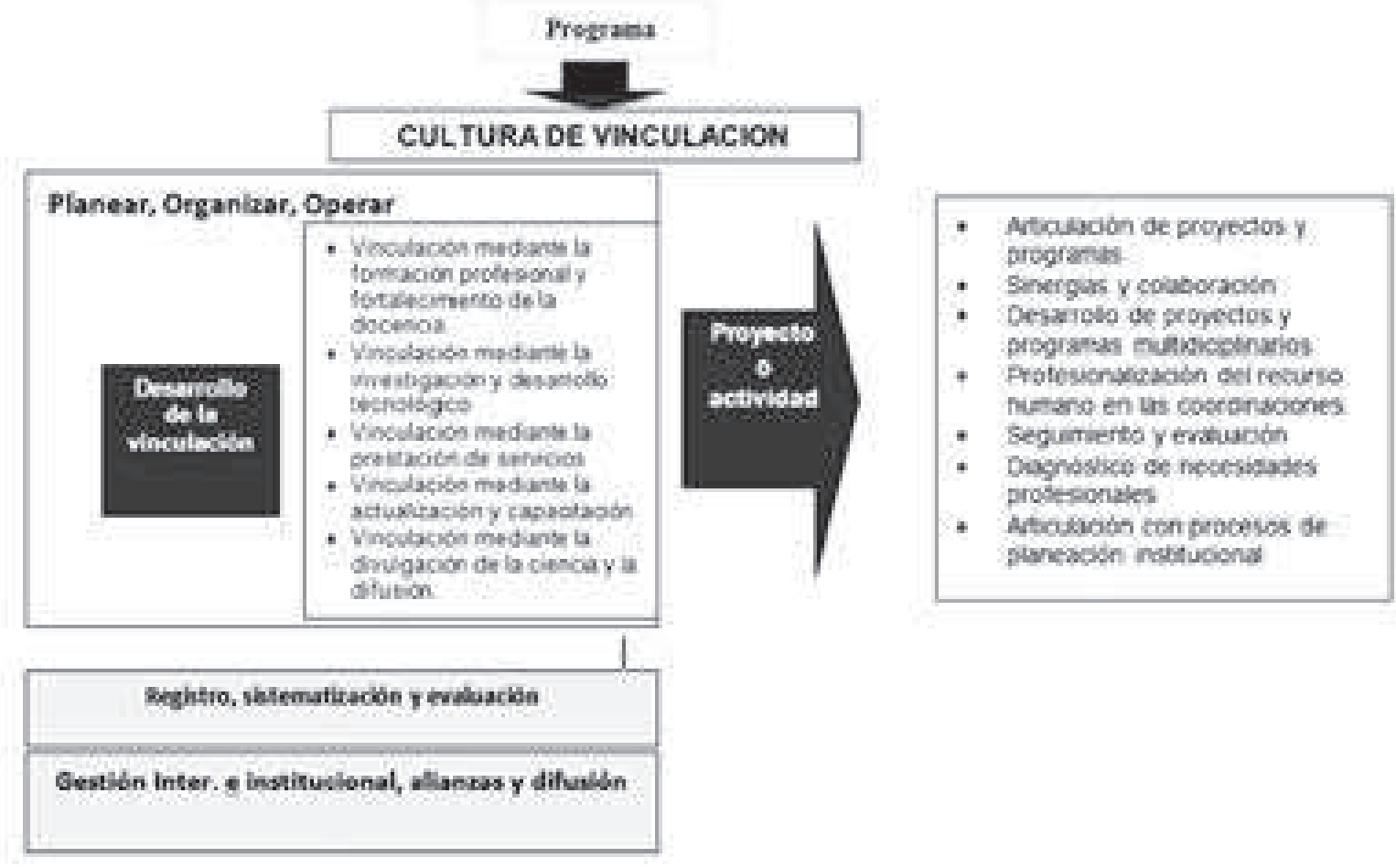

Nota: Adaptado del modelo de vinculación U. Veracruzana (2006).

Desde la adaptación del modelo de vinculación de la Universidad Veracruzana (2006), se presenta en la figura 10 un acercamiento esquemático de una posible adopción de esta estrategia en la carrera de estudio. Dentro de los aspectos que se contemplarían son un plan de desarrollo del personal académico; planeación de procesos; registro, sistematización y evaluación de los procesos de docencia, de investigación y de extensión. Asimismo la promoción y vinculación de la carrera en estudio con los diferentes sectores que conforman la sociedad costarricense.

4) Implementación de injerencia académica en la estructura funcional existente dentro de las escuelas, carreras y cátedras. Es 
necesario establecer vínculos de acción que permiten articular el nivel Macro (carrera en estudio) con el nivel Micro (cátedras). Las cátedras son dependencias que actualmente colaboran bajo un estilo funcional matricial. Para una planeación conjunta se recomienda una nueva acción en la organización funcional: la injerencia directa académica del nivel Macro con el Micro (véase figura 11).

\section{Figura 11. Organización funcional actual y propuesta en el nivel Mega, Macro y Micro.}

Situación Actual Funcional

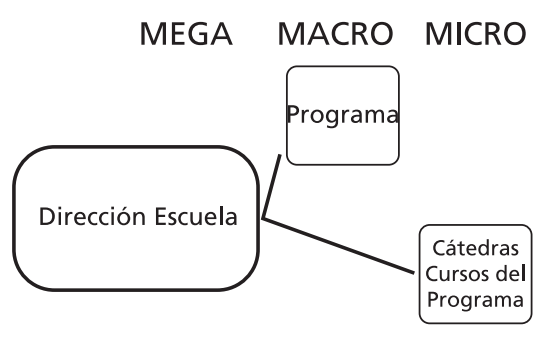

Situación Funcional Sugerida

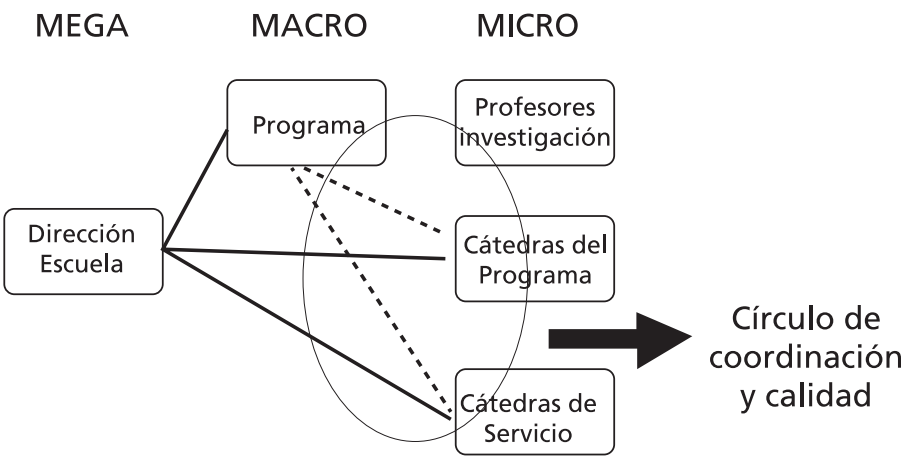

Nota: La línea intercalada significa autoridad del Programa sobre las Cátedras educativa, esto es sobre los cursos que integran la carrera. No es una línea de coordinación, sino sobre el diseño curricular, la implementación de los cursos y su control (injerencia académica).

Paralelamente con el cambio sugerido en la figura 11, es importante integrar las relaciones necesarias del personal académico con el desarrollo del currículo, el programa y el proceso de enseñanza y aprendizaje (véase figura 12). El plan de estudios del programa y el diseño curricular de los cursos deben ser pertinentes a las necesidades de la sociedad, a quien responde el desarrollo de la carrera como tal. Para ello es necesario un plan de desarrollo de personal académico que responda a los requerimientos de calidad institucional como respaldo de los procesos de enseñanza y aprendizaje. 
Figura 12. Relación del personal académico con el currículo, el programa y el proceso de enseñanza y aprendizaje

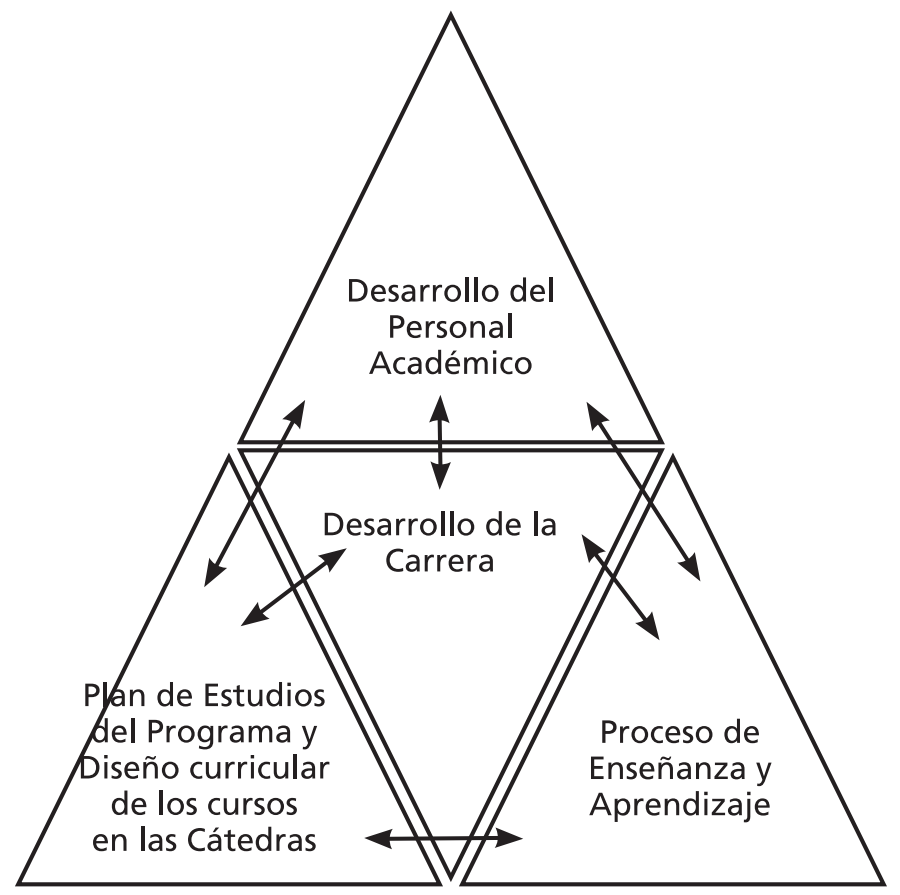

Nota: Adaptado de Azze, (2009).

En cuanto al uso de la tecnología en la educación a distancia es una de las tendencias que debe contemplarse primeramente en la misión de la UNED (Mega) para que así sea reflejado en los niveles subsiguientes $($ Macro $=$ Carreras, Micro $=$ Cátedras y el nivel operativo $)$. Lo anterior conllevaría a contemplar el uso de las TIC, como una realidad existente en la UNED, lo cual no ha sido reflejado expresamente en el modelo pedagógico.

Finalmente, se recomienda para futuros estudios realizar investigaciones de la gestión académica superior universitaria para conocer la correlación de las declaraciones de la misión, visión con sus modelos pedagógicos, curriculares y didácticos. 


\section{Referencias bibliográficas}

Azze, A. (2009). Debilidades comunes de las carreras autoevaluadas en la Escuela de Ciencias Exactas y Naturales: Posibles causas y niveles institucionales para la solución. (Sin publicar). Programa de Autoevaluación y Acreditación de la Universidad Estatal a Distancia. San José, Costa Rica: UNED.

Escuela de Ciencias Exactas y Naturales. (2008). Plan Estratégico 2008-2012- ECEN. [Folleto] San José, Costa Rica: UNED.

Kaufman, R., Watkins, R., y Leigh, D. (2001). Useful educational results: Defining, prioritizing and accomplishing. Lancaster, PA: Proactive Publishing.

Ulate, R. (2009). Análisis de Sistema: Programa de Protección y Manejo de Recursos Naturales. (Sin publicar). Curso Introducción a Diseño de Sistemas Instruccionales. San José, Costa Rica: NOVA.

Universidad Estatal a Distancia (2004). Modelo Pedagógico. Recuperado de http://www.uned.ac.cr/paa/pdf/Materiales-autoev/24.pdf

Universidad Estatal a Distancia (2008). Informe de autoevaluación Programa de Protección y Manejo de Recursos Naturales. [Informe de solicitud de acreditación ante el Sistema Nacional de Acreditación de la Educación Superior]. Costa Rica: UNED.

Universidad Veracruzana (2006). Planeación de la vinculación: Red de coordinadores de vinculación. Hacia una nueva cultura de vinculación. Dirección de Vinculación General. México. Recuperado de www.uv.mx/vincula/servicios/social/brigadas.htm 